\title{
DISTANCE AND DISSIMILARITY
}

\begin{abstract}
This paper considers whether an analogy between distance and dissimilarity supports the thesis that degree of dissimilarity is distance in a metric space. A traditional way to justify the thesis would be to prove representation and uniqueness theorems, according to which if comparative dissimilarity meets certain qualitative conditions, then it is representable by distance in a metric space. But I will argue that those qualitative conditions which are strong enough to capture the analogy between distance and dissimilarity are not met by either actual or possible particulars.
\end{abstract}

\section{INTRODUCTION}

Imagine things arranged in space so that the more similar they are, the closer they are together. Then it is plausible that the degree of dissimilarity between those things is their distance apart in that space. If the merchandise in a department store, for example, were arranged so that more similar items were closer together, then the degree of dissimilarity between the shoes and the socks, for example, would be roughly proportional to the distance between the shoe section and the sock section. This paper considers to what extent this analogy between distance and dissimilarity supports the thesis that degree of dissimilarity is distance in a metric space.

The thesis that degree of dissimilarity is distance in a metric space is not trivial. The produce in a grocery store, for example, is not quite arranged so that more similar goods are closer together - despite their dissimilarity, milk and bread, for example, are often together at the back of the store. Perhaps this problem is due merely to greed on the part of grocers, or a limitation of the physical geometry of the store. But perhaps it is impossible even in

Date: March 2, 2018. 
principle to arrange the store so that similar goods are closer together. So, despite the suggestiveness of the analogy between distance and dissimilarity, the thesis that degree of dissimilarity is distance in a metric space requires a more thorough justification.

A traditional way to justify the thesis that degree of dissimilarity is distance in a metric space is via the proof of a representation and uniqueness theorem. According to a representation theorem, if a structure meets certain qualitative conditions, then it has a certain quantitative representation - if goodness of films, for example, met certain qualitative conditions, then it would be possible to represent how good they are on a five star scale. Likewise, it is possible to prove a representation theorem to the effect that if comparative dissimilarity meets certain qualitative conditions, then it is representable by distance in a metric space.

In addition to a representation theorem, it is desirable to prove a uniqueness theorem, according to which all alternative representations of a given structure are a certain kind of transformation of each other. A uniqueness theorem for the representation of temperature, for example, would show that the representation in terms of degrees Celsius is unique, since any alternative representations of temperature - such as in terms of degrees Fahrenheit or degrees Kelvin - are merely affine transformations of the representation in terms of degrees Celsius.

In particular, if a structure is represented by an ordinal scale, then any other ordinal scale - in other words, any scale preserving the same order representing that structure is an increasing function of the first [5, 11]. If goodness of films, for example, is representable on a five star scale, then a ten star scale would be equally as good, as long as it represents the films in the same order. But I will argue in section (3) that representations of degree of dissimilarity derived from an ordinal scale fail to capture the spirit of the analogy between distance and dissimilarity. 
If a structure is represented by a ratio scale, on the other hand, then any other ratio scale representing that structure is a positive multiple of the first - in other words, the ratio of two measurements will be the same according to every representation [5, 10]. Whereas an ordinal scale represents only whether a given quantity is greater than another, a ratio scale represents how much greater, since the representation of any two quantities will be in the same proportion to each other according to any ratio scale. If one quantity is, for example, twice as great as another according to one ratio scale, then it will be twice as great according to every ratio scale.

If enough things are arranged in space so that the more similar they are, the closer they are together, then it's plausible not only that their degree of dissimilarity is their distance apart in that space, but also that if two things are twice as far in that space from each other as two other things are, then the first two things are twice as dissimilar to each other as the second two things are. So the analogy between distance and dissimilarity suggests that the representation of comparative dissimilarity by distance in a metric space is a ratio scale. I will concede in sections (4) and (5) that a representation of degree of dissimilarity based on a ratio scale would succeed in capturing the spirit of the analogy between distance and dissimilarity.

Nevertheless, I will present a dilemma for the thesis that degree of dissimilarity is representable as distance in a metric space. If comparative dissimilarity is a relation between actual particulars, then even if it meets the conditions required for an ordinal scale, it might not meet those required for a ratio scale. But if comparative dissimilarity is a relation between possible particulars, then although it meets one of the two important conditions for representability by a ratio scale, it fails to meet the other. So the representation theorem fails to show that comparative dissimilarity is representable by a ratio scale.

A closely related technical reason that possible particulars may not be representable by distance in a metric space has been pointed out before 
(see especially [7, 51] and [15, 458-9]). But in the context of an ordinal scale, it is natural to dismiss this problem as a mere technicality: as David Lewis, for example, writes "This limitation hardly seems serious" [7, 51]. In contrast, I will argue that in the context of representation by a ratio scale, the problem is not merely technical, but seriously undermines the justification of the thesis that comparative dissimilarity is representable by distance in a metric space.

The analogy between distance and dissimilarity has many philosophical applications, including to possible worlds semantics for conditionals [7, 5052], the analysis of probability [1] [2], the measurement of likeness to truth [9, 1-17][10, 34-38], and the philosophy of modality generally [12, 352]. Not every application of the analogy requires the more exact thesis that degree of dissimilarity is distance in a metric space - possible worlds semantics for conditionals, for example, does not require dissimilarity to be measurable by numerical degrees [7, 50-52]. But some applications do - for example, the analysis of numerical degree of probability in terms of numerical degree of similarity [2, 461].

A particularly important application of numerical degrees of dissimilarity is in the explanation of how overall similarity can be determined by weighing up similarity in various respects (see especially [6, 459-62]). But the representation and uniqueness theorems relevant to this application are considerably more complex than those discussed here [13, 175-99]. Moreover, it is only under special conditions that they support the thesis that degree of dissimilarity is distance in a metric space, and thus the analogy between distance and dissimilarity [13, 186-7]. So I will not address this application in this paper.

Regardless of its applications, the analogy between distance and dissimilarity, and the thesis that degree of dissimilarity is distance in a metric space that it supports, is interesting not only for its applications, but also 
in its own right. The nature of similarity is as central a topic in philosophy as, for example, the nature of probability. So the representation and uniqueness theorems purporting to show that degree of dissimilarity is distance in a metric space should be as widely discussed as those purporting to show that credences satisfy the probability calculus. The discussion of similarity should not be confined to digressions in work on counterfactuals or truthlikeness, but should also be pursued for its own sake.

The representation and uniqueness theorems discussed in this paper are extremely familiar (especially from [13, 159-225]), but their relevance to the metaphysics of properties and resemblance, and so also their relevance to applications of the thesis that degree of dissimilarity is distance in a metric space to problems in metaphysics and philosophy generally, has not been very widely noticed. I will argue that some conditions which may seem like harmless idealisations in applications to psychology and the social sciences, are in fact deeply controversial in the context of metaphysics, and so cannot be presupposed in philosophy generally.

\section{Metric Spaces}

The analogy between distance and dissimilarity motivates the thesis that degree of dissimilarity is distance in a metric space, where:

Definition 1. [13, 46] A metric space is an ordered pair $\langle A, \delta\rangle$ of a set $A$ and a distance function $\delta: A \times A \rightarrow \mathbb{R}$ such that:

(1) $\delta(a, a)=0$ (minimality)

(2) $\delta(a, b)>0$ if $a \neq b$ (non-colocation)

(3) $\delta(a, b)=\delta(b, a)$ (symmetry)

(4) $\delta(a, b)+\delta(b, c) \geq \delta(a, c)$ (the triangle inequality).

In space, for example, (1) every point is at distance zero from itself, (2) all distinct points are at a positive distance from each other, (3) the distance from point $a$ to point $b$ is the same as the return distance from point $b$ to $a$, 
and (4) the distance from point $a$ to point $c$ via point $b$ is always at least as great as the distance from point $a$ directly to point $c$.

A simple example of a metric space is distance between the points on the real line, or the set of real numbers $\mathbb{R}$ and their absolute difference $\delta(i, j)=$ $|i-j|$. A more paradigmatically spatial example is three-dimensional Euclidean space, or the set of triples $i=\left\langle i_{1}, i_{2}, i_{3}\right\rangle \in \mathbb{R}^{3}$ and their Pythagorean distance $\delta(i, j)=\sqrt{\left(i_{1}-j_{1}\right)^{2}+\left(i_{2}-j_{2}\right)^{2}+\left(i_{3}-j_{3}\right)^{2}}$. In general, the $n$ dimensional Euclidean space $\mathbb{R}^{n}$ is a metric space, and a natural choice for representing overall comparisons of dissimilarity which depend on dissimilarity in $n$ different respects.

Another paradigmatic example is the unit circle with it intrinsic metric, or the distance around the arc connecting two points (in other words, the set of pairs $i=\left\langle i_{1}, i_{2}\right\rangle \in \mathbb{R}^{2}$ such that $\sqrt{i_{1}^{2}+i_{2}^{2}}=1$ and their intrinsic distance $\left.\delta(i, j)=\left|\arcsin j_{2}-\arcsin i_{2}\right|\right)$. The unit circle with its intrinsic metric contrasts with the unit circle with its Euclidean metric, or the distance across the chord connecting two points (in other words, the set of pairs $i=\left\langle i_{1}, i_{2}\right\rangle \in \mathbb{R}^{2}$ such that $\sqrt{i_{1}^{2}+i_{2}^{2}}=1$ and their Pythagorean distance $\left.\delta(i, j)=\sqrt{\left.\left(i_{1}-j_{1}\right)^{2}+\left(i_{2}-j_{2}\right)^{2}\right)}\right)$. So the thesis that degree of dissimilarity is measurable by distance in a metric space is compatible with many theses about the geometry of that space.

Nevertheless, the conception of distance embodied in the definition of a metric space is too general to capture the analogy between distance and dissimilarity, because many metric spaces lack the spatial character of the paradigmatic examples. An extreme case is the discrete metric, which may be defined on any set by stipulating that the distance between any element and itself is zero, and the distance between any two distinct elements is one. If it turned out that everything was equally dissimilar to everything else, but nothing was at all dissimilar to itself, then degree of dissimilarity could be represented by the discrete metric. But this representation would hardly capture the analogy between dissimilarity and distance. 
So the analogy between distance and dissimilarity suggests not only that degree of dissimilarity is distance in a metric space, but also that that metric space should meet two further characteristically spatial conditions. The first condition is stated in terms of betweenness, where:

Definition 2. A point $b$ is between points $a$ and $c$ in a metric space $\langle A, \delta\rangle$ if and only if $\delta(a, b)+\delta(b, c)=\delta(a, c)$.

In other words, a point $b$ is between points $a$ and $c$ if and only if the distance from point $a$ to $c$ via $b$ is equal to the distance from point $a$ directly to point $c$, or, in other words again, $b$ lies on the shortest path from $a$ to $c$.

Since in a discrete metric space the distance between any two distinct elements is one, no distinct elements in the discrete metric space have any element in between them. In a dense metric space, on the other hand, all distinct elements have an element between them.

Definition 3. A metric space $\langle A, \delta\rangle$ is dense if and only if between any two distinct points $a, c \in A$ there is a distinct point $b \in A[3$, 40].

Euclidean space, for example, is dense, since between any two distinct points in Euclidean space lie the points on the line segment connecting them. Likewise, between any two distinct points on the unit circle with its intrinsic metric, for example, lie the points on the arc of the circle connecting them. The unit circle with its Euclidean metric, in contrast, is not dense, since the chord connecting two points across the circle lies outside the space. Likewise, the discrete metric on a set is obviously not dense.

The second characteristically spatial condition suggested by the analogy with distance is completeness:

Definition 4. A metric space $\langle A, \delta\rangle$ is complete if and only if for every sequence of elements $a_{1}, \ldots, a_{n}, \ldots$ in $A$ such that the distance between successive pairs $\delta\left(a_{1}, a_{2}\right), \delta\left(a_{2}, a_{3}\right), \ldots, \delta\left(a_{n}, a_{n+1}\right), \ldots$ converges to zero, there is 
an element $a \in A$ such that the distance $\delta\left(a_{1}, a\right), \delta\left(a_{2}, a\right), \ldots, \delta\left(a_{n}, a\right), \ldots$ between successive elements and $a$ also converges to zero (where a sequence of real numbers $r_{1}, r_{2} \ldots, r_{n}, \ldots$ converges to zero if and only if for any $\epsilon>0$ there is an $m$ such that $r_{l}<\epsilon$ for all $l>m$ ) [3, 28].

In other words, if there is a sequence of elements all but finitely many of which are within an arbitrarily small distance of each other, then there is a particular element which all but finitely many are within an arbitrarily small distance of.

In Euclidean space, every pair of distinct points are joined by a line segment consisting of the points in between them. Density and completeness are important properties of a metric space because they entail that every pair of distinct points in the space are joined by a metric segment, a subset of the space isometric to a Euclidean line segment (where subsets of two metric spaces are isometric if and only if there is a bijective distance-preserving function between them) [3, 40]. The arc between each pair of distinct points on the unit circle with its intrinsic metric, for example, is isometric to a straight Euclidean line segment of the same length.

By generalising the fact that any two points in Euclidean space are joined by a line segment, the existence of metric segments encapsulates paradigmatically spatial characteristics of many metric spaces. So in addition to considering whether the analogy between distance and dissimilarity supports the thesis that degree of dissimilarity is distance in a metric space, it is also worth considering whether that metric space is dense and complete. I will argue in section (5) that although the qualitative conditions on comparative dissimilarity corresponding to density and completeness suffice to capture the analogy between distance and dissimilarity, they also exacerbate its problems. 


\section{Ordinal Scales}

A traditional way to justify the thesis that degree of dissimilarity is distance in a metric space is to prove a representation theorem, according to which if a structure meets certain qualitative conditions, then it is representable by distance in a metric space, and then to argue that (possible) particulars under the relation of comparative dissimilarity meet those conditions. In the discussion below, the conditions are stated for an arbitrary set $A$ and quaternary relation $\succsim$, but the intended interpretation is that $A$ is the set of (possible) particulars and $a b \succsim c d$ is the relation of $a$ being at least as dissimilar to $b$ as $c$ is to $d$ (as usual, $a b \sim c d$ abbreviates $a b \succsim c d$ and $c d \succsim a b ; a b \succ c d$ abbreviates $a b \succsim c d$ but not $c d \succsim a b)$.

(Note that some discussions are conducted in terms of a ternary or threeplace relation such that $b \succsim_{a} c$ is interpreted as meaning that $b$ is at least as similar to $a$ as $c$ is (see for example [7, 48-50]). The ternary relation is definable in terms of the quaternary relations, since $b$ is as similar to $a$ as $c$ is if and only if $a$ and $b$ are as similar as $a$ and $c$ or, in other words, $b \succsim_{a} c$ if and only if $b a \succsim c a$. The quaternary relation, in contrast, cannot in general be defined in terms of the ternary relation. So it is the quaternary relation which is the more appropriate primitive [15].)

The following definition gives simple qualitative necessary conditions for a structure $\langle A, \succsim\rangle$, such as (possible) particulars under the relation of comparative dissimilarity, to be representable by a distance function:

Definition 5. [13, 161] The structure $\langle A, \succsim\rangle$ of a set $A$ and a quaternary relation $\succsim$ on $A \times A$ is a proximity structure if and only if for all $a, b, c, d, e, f \in$ $A$ the following conditions hold:

(1) $\succsim$ is a weak ordering, or in other words:

(a) Either $a b \succsim c d$ or $c d \succsim a b$ (connectedness)

(b) if $a b \succsim c d$ and $c d \succsim e f$ then $a b \succsim e f$ (transitivity)

(2) $a a \sim b b$ (minimality) 
(3) $a b \succ a a$ if $a \neq b$ (identity of indissimilars)

(4) $a b \sim b a$ (symmetry).

If comparative dissimilarity, for example, is representable by a distance function, then it is necessary that the set of (possible) particulars under the relation of comparative dissimilarity be a proximity structure.

Condition (1), weak ordering or connectedness and transitivity, is necessary for comparative dissimilarity to be representable by any function to the real numbers at all - since the relation $\geq$ of being at least as great as between real numbers is connected and transitive, any relation it represents must be connected and transitive as well. (For criticism of connectedness see [4, 38-39] [8, 480-481]; for defence see [6, 462-464]. For criticism of transitivity, see [15, 463] and [8, 475-476]. Weak ordering is a presupposition of the application to counterfactuals - see [7, 48].)

The remaining three conditions of the definition of a proximity structure correspond to the first three conditions in (1), the definition of a metric space, except the former are qualitative whereas the latter are quantitative. However, note that there is no qualitative condition in the definition of a proximity structure corresponding to the triangle inequality, condition (4) in the definition of a metric space, for a reason that will be explained below. (For criticism of minimality, symmetry, and also the triangle inequality see [14, 328-329]. Minimality is a presupposition of the application to counterfactuals [7, 48], but symmetry is not [7, 51].)

Condition (3), the identity of indissimilars, is controversial, because it is closely related to the identity of indiscernibles, according to which if $a$ and $b$ are not identical, then $a$ has different properties from $b$ : supposing that $a$ is more dissimilar to $b$ than $c$ is to $d$ if and only if $a$ has more properties not in common with $b$ than $c$ has not in common with $b$, then they are equivalent [15, 463-4]. So if there are distinct particulars which share all of their properties, and so are exactly alike, then neither the identity of indiscernibles nor the identity of indissimilars is satisfied. 
But this controversy can be circumvented in the presence of the other conditions. Consider the relation of being as dissimilar to a particular as it is to itself, or $x y \sim y y$. Minimality entails that that relation is reflexive, since it entails every particular is as similar to itself as it is to itself. And minimality, symmetry and transitivity entail that it is symmetric and transitive. So it follows that the relation of being as dissimilar to a particular as it is to itself is an equivalence relation, which partitions things into equivalence classes of exactly alike particulars [13, 47].

If a pair of equivalence classes are defined as being at least as dissimilar to each other as a second pair if and only if two representative particulars chosen from each of the first pair are at least as dissimilar to each other as two chosen from each of the second pair, then the set of equivalence classes of exactly alike particulars under this derived relation of comparative dissimilarity is a proximity structure, since it satisfies condition (3), and the reasoning below applies to the derived relation of comparative dissimilarity between equivalence classes of exactly alike particulars.

Since the relation $\sim$ is itself reflexive, symmetric and transitive, it's also possible to divide $A \times A$ into equivalence classes of equally dissimilar pairs $A \times A / \sim$ with a derived order, $B \succsim C$ if and only if $a b \succsim c d$ for all $a, b \in B$ and $c, d \in C$. So it's possible to reduce the problem of finding a representation of the weakly ordered set $\langle A \times A, \succsim\rangle$ to the problem of finding a representation for the totally ordered set $\langle A \times A / \sim, \succsim\rangle$. In other words, it's possible to reduce the problem of representing degree of dissimilarity between particulars to the problem of finding a representation of degree of dissimilarity between equivalence classes of equally dissimilar pairs.

A necessary and sufficient condition for a simply ordered set such as the set of equivalence classes of equally dissimilar pairs under their derived order to be representable by an ordinal scale is that it have a countable order-dense subset, where: 
Definition 6. [5, 40] If $\langle B, \succsim\rangle$ is a simple order and $C$ is a subset of $B$, then $C$ is order-dense in $B$ if and only if for all $a, c \in B$ such that $a \succ c$ there exists $b \in C$ such that $a \succsim b \succsim c$.

The paradigm case of definition (6) is the countable order-density of the rational numbers in the reals, since the rational numbers are a countable subset of the real numbers, and since between every two distinct real numbers there is a rational number.

Countable order-density is sufficient for a totally ordered set to be represented by an ordinal scale, since each element of the countable subset can be represented by a rational number, and then each element not in the countable subset can be represented by the least upper bound of the rational numbers of the elements below it. And countable order-density is necessary for a totally ordered set to be represented by an ordinal scale, since the elements represented by the rational numbers must be countably order-dense in the elements represented by the real numbers [5, 40-42].

The definition of a proximity structure (5) together with (6) the definition of countable order-density allow the statement of the following representation theorem:

Theorem 1. [13, 162] The structure $\langle A, \succsim\rangle$ of a set $A$ and a quaternary relation $\succsim$ on $A \times A$ is representable by a distance function in a metric space if and only if $\langle A, \succsim\rangle$ is a proximity structure and $A \times A / \sim$ has a countable order-dense subset.

In particular, if $\langle A \times A / \sim, \succsim\rangle$ has a countable order-dense subset, then it is representable by an ordinal scale which also represents $\langle A \times A, \succsim\rangle$, and from which it is possible to derive a distance function.

In order to derive a distance function from the ordinal scale representing $\langle A \times A, \succsim\rangle$, it's necessary only to ensure it satisfies condition (4), the triangle inequality, since the other three conditions of the definition of a metric space are guaranteed by the corresponding qualitative conditions in the definition 
of a proximity structure. That may be achieved by defining the degree of dissimilarity between each particular and itself as zero, and rescaling all the other degrees of dissimilarity to be between one and two, so that the sum of all the degrees of dissimilarity between distinct particulars will always be greater than two, and the condition $\delta(a, b)+\delta(b, c) \geq \delta(a, c)$ will always be met [13, 162][15, 462].

Unfortunately, the justification of the thesis that degree of dissimilarity is representable by distance in a metric space encapsulated in this theorem has two problems with infinity. The first problem is that if there are more equivalence classes of equally dissimilar pairs than there are real numbers, then comparative dissimilarity can't be represented by a function to the real numbers, since then some unequally dissimilar pairs would have to be represented by the same real numbers. If there are more equivalence classes of equally dissimilar pairs than real numbers, then there are simply too many degrees of dissimilarity to be represented by real numbers (just as there are too many unequally good films to be represented by only five stars).

Even if there are only as many equally dissimilar pairs as there are real numbers, comparative dissimilarity may not be representable by a function to the real numbers, since not every ordered set of continuum-many elements has a countable order-dense subset. Consider, for example, the coordinates of the plane $\left\langle i_{1}, i_{2}\right\rangle \in \mathbb{R}^{2}$ under their lexicographic ordering, $\left\langle i_{1}, i_{2}\right\rangle \succsim\left\langle j_{1}, j_{2}\right\rangle$ if and only if either $i_{1}<j_{1}$ or else both $i_{1}=j_{1}$ and $i_{2} \leq j_{2}$. Since every order-dense subset of the plane under the lexicographic ordering must contain at least one distinct element on each horizontal line, and there are as many horizontal lines as real numbers, every order-dense subset of the plane under the lexicographic ordering must contain as many elements as real numbers and so uncountably many elements. So the lexicographically ordered plane has no countable order-dense subset.

This is not a problem if the number of particulars is finite or countable, since in that case the number of equally dissimilar pairs is also finite or 
countable, and so is trivially an order-dense subset of itself. If the number of particulars is continuum many, then it's possible that the number of equally dissimilar pairs of particulars is also continuum many. And it is still not a problem if the equally dissimilar pairs of particulars under the order of comparative dissimilarity are isomorphic to a subset of the real numbers in their usual ascending order. But even if none of these favourable conditions obtain, countable order-density seems like a harmless idealisation. As David Lewis writes, "this limitation hardly seems serious" [7, 51].

The second problem is that the triangle inequality was obtained so easily only because the numbers chosen preserve only the order of the degrees of dissimilarity they represent, and so could be rescaled arbitrarily - the proportions between the numbers were irrelevant [15, 462]. If $a$ is more dissimilar to $b$ than $c$ is to $d$, for example, then the degree of dissimilarity of $a$ to $b$ must be greater than that of $c$ to $d$, but how much greater is completely

arbitrary. If the degree of dissimilarity of $c$ to $d$ is $\frac{3}{2}$, for example, the degree of dissimilarity of $a$ to $b$ could be any real number strictly between $\frac{3}{2}$ and 2 at all, no matter how similar or dissimilar $a$ and $b$ are.

So the justification encapsulated in the ordinal representation theorem fails to capture the analogy between distance and dissimilarity. The source of the problem is that the distance function representing comparative dissimilarity is derived from an ordinal scale, which represents only which pairs of particular are as or more dissimilar to others, but not how much more or less dissimilar they are than others. If dissimilarity is truly analogous to distance, it should be represented by a ratio scale, which would represent not only which things are more similar to each other, but also by how much.

\section{Ratio Scales}

In order to capture the analogy between distance and dissimilarity, it is desirable to find a representation of comparative dissimilarity which represents not only whether one pair are more dissimilar to each other than 
another pair are, but by how much. In the measurement of length, for example, it is possible not only to check that one rod is longer than another, but also how much longer, by concatenating rods together - if the concatenation of two rods of equal length, for example, are the same length as a third rod, then we can conclude not only that the first rod is longer than the other two, but that it is twice as long.

Dissimilarities cannot be concatenated like rods can. But if dissimilarity is analogous to distance, then we can think of some particulars as being between each other, in such a way that the dissimilarity between a pair of particulars can be calculated by adding up the dissimilarities of the particulars which lie between them. Suppose, for example, that venison is as dissimilar from beef as kangaroo is from venison, and moreover that venison is between beef and kangaroo. Then one can conclude that the dissimilarity between beef and kangaroo is twice as great as the dissimilarity between beef and venison.

In section (2), betweenness was defined quantitatively in terms of distance or degree of dissimilarity. But in order to underwrite the justification of the analogy between distance and dissimilarity, betweenness must be defined qualitatively in terms of merely comparative similarity, as in the following definition:

Definition 7. [13, 164] If $A$ is a set and $\succsim$ is a quaternary relation on $A$, then for all $a, b, c \in A$ the ternary relation ( $a b c\rangle$ holds if and only if for all $d, e, f \in A$ :

(1) if $a b \succsim d e$ and $d f \succsim a c$, then $e f \succsim b c$, and

(2) if either of the inequalities in the antecedent of (1) is strict, so is the inequality in the consequent.

The betweenness relation $\langle a b c\rangle$ holds if and only if the ternary relations $\langle a b c$ ) and $(c b a\rangle$ both hold. 
The definition is motivated by the analogy with spatial distance. Suppose, for example, that Abu Dhabi is between Sydney and London. Then if there are two cities more distant than Sydney and London - say Melbourne and Edinburgh - and a city closer to Melbourne than Abu Dhabi is to Sydney say Singapore - then the distance from Singapore to Edinburgh must exceed the distance from Abu Dhabi to London. Otherwise the possibility of flying further - from Melbourne to Edinburgh via Singapore - via a shorter path would show the route from Sydney to London via Abu Dhabi is not a shortest path, and thus that Abu Dhabi is not between Sydney and London.

The conditions in (5), the definition of a proximity structure, are sufficient for the degrees of dissimilarity between particulars to be the sum of the dissimilarities of the particulars between them in combination with the conditions in the following definition:

Definition 8. [13, 168] A proximity structure $\langle A, \succsim\rangle$ is segmentally additive if and only if for all $a, b, c, d \in A$ :

(1) if $a b \succsim c d$, then there is an $e \in A$ such that $a e \sim c d$ and $\langle a e b\rangle$ (segmental solvability)

(2) if $c \neq d$ then there is a sequence $e_{0}, \ldots, e_{n} \in A$ such that $e_{0}=a$, $e_{n}=b$ and $c d \succsim e_{i} e_{i+1}$ for all $i<n$ (the Archimedean condition).

If the set of particulars under comparative dissimilarity, for example, is a segmentally additive proximity structure, then comparative dissimilarity is representable by a ratio scale.

According to condition (1), segmental solvability, whenever two particulars $a$ and $b$ are at least as dissimilar as $c$ and $d$, there's a particular $e$ between $a$ and $b$ such that $a$ and $e$ are just as dissimilar as $c$ and $d$. Although beef is more dissimilar to kangaroo than chicken is to duck, for example, segmental solvability requires that there is a meat - say, venison - which is between beef and kangaroo, and which is exactly as dissimilar to beef as chicken is to duck. 
The idea behind segmental solvability is that the numerical degree of dissimilarity between two particulars can be measured by counting the degrees of dissimilarity of the particulars between them. If between beef and whale, for example, there are at least three particulars - venison, buffalo and kangaroo - as dissimilar from each other as chicken is to duck, then we could conclude that the degree of dissimilarity between beef and whale is at least four times the degree of dissimilarity between chicken and duck.

According to (2), the Archimedean condition, for any two particulars $a$ and $b$, and any two different particulars $c$ and $d$, there is a sequence of particulars beginning with $a$ and ending with $b$, each successive pair of which are at least as similar to each other as $c$ is to $d$. No matter how much gamier pterodactyl is than chicken, for example, there should be a sequence of meats - say from chicken, to quail, to turkey, to emu, ... to pterodactyl - such that each successive pair in this sequence are at least as similar to each other as mutton is to goat.

The idea behind the Archimedean condition is that nothing is so distant that it cannot be reached by a finite number of small steps. Even though my commute to work, for example, is very short, there is nowhere on earth that I cannot reach by taking a journey each leg of which is at least as short as my commute to work. So whereas segmental solvability ensures that between any points on my journey there are stops close enough for me to travel to, the Archimedean condition ensures that my destination is not so far away that I cannot reach it after passing only finitely many stops.

Together segmental solvability and the Archimedean condition entail that for any particulars $a$ and $b$ and any two different particulars $e$ and $f$, there is a sequence of particulars between $a$ and $b$ each successive pair of which are just as dissimilar to each other as $e$ and $f$. How much more dissimilar $a$ is to $b$ than $c$ is to $d$ can then be approximated by how many more particulars there are in the sequence between $a$ and $b$ than in the sequence between $c$ 
and $d$, or in other words as the ratio of the length of the sequence between $a$ and $b$ to the length of the sequence between $c$ and $d$.

The more similar $e$ is to $f$, the better the approximation, and in the limit, or else if no different particulars are more similar than $e$ and $f$, it is exact. So together segmental solvability and the Archimedean condition entail the following theorem:

Theorem 2. [13, 168] If $\langle A, \succsim\rangle$ is a segmentally additive proximity structure, then there exists a function $\delta: A \times A \rightarrow \mathbb{R}$ such that:

(1) $\langle A, \delta\rangle$ is a metric space

(2) $a b \succsim c d$ if and only if $\delta(a, b) \geq \delta(c, d)$

(3) $\langle a b c\rangle$ if and only if $\delta(a, c)=\delta(a, b)+\delta(b, c)$ (additivity)

(4) If $\langle A, \gamma\rangle$ also satisfies (1)-(3), then $\gamma(a, b)=r \delta(a, b)$ for some $r>0$. (uniqueness)

Whereas the first two clauses of theorem (2) reassert the content of theorem (1) - that there is a representation of degree of dissimilarity as distance in a metric space - the third clause asserts that there is a representation in which the degrees of dissimilarity are additive, and the fourth clause asserts that these properties are unique to representations in which degrees of dissimilarity are in the same ratio to each other.

Unlike the representation derived from an ordinal scale in section (3), the ratio scale established by theorem (2) establishes not only that the dissimilarities between pairs of particulars must have a certain order, but also that they must have certain proportions. Suppose, for example, that there are just three particulars $a, b$ and $c$, and $a$ is as dissimilar to $b$ as $b$ is to $c$, but $a$ is more dissimilar to $c$ than $a$ is to $b$. Then it follows from (7) the definition of betweenness that $b$ is between $a$ and $c$, and so the dissimilarity between $a$ and $c$ must be exactly twice the dissimilarity between $a$ and $b$.

Nevertheless, like the representation derived from an ordinal scale, the justification of the analogy between distance and dissimilarity encapsulated 
in theorem (2) still has two problems with infinity. To see the source of the first problem with infinity, note that the definition (8), of a segmentally additive proximity structure, and the statement of theorem (2) omit any mention of countable order-density. This is not because countable orderdensity is not a necessary condition of the theorem, but because countable order-density is entailed by the definition of a segmentally additive proximity structure.

In the context of an ordinal scale the possibility that the countable orderdensity condition may not be met hardly seemed serious. But in the context of a ratio scale, the segmental solvability and the Archimedean condition face a dilemma. On the one hand, if the quaternary relation of comparative dissimilarity is interpreted as a relation between actual particulars, then the condition of segmental solvability is not met. On the other hand, if comparative dissimilarity is interpreted as a relation between merely possible particulars, then the Archimedean condition is not met.

If comparative dissimilarity is interpreted as a relation between actual particulars, segmental solvability is not met because there are some pairs of dissimilar particulars which don't have any distinct particular between them, even though there are other pairs of particulars which are not so dissimilar as they are. Turkey, for example, is more dissimilar to duck than beef is to venison. But there is no actual meat between turkey and duck which is just as dissimilar to turkey as beef is to venison (tastes may vary, but you can substitute your own example).

Segmental solvability is not a plausible condition for actual particulars, but it is more plausible for possible particulars, since it is more plausible that for every pair of dissimilar particulars, there are possible particulars between which are just as dissimilar as any pair of less dissimilar particulars are to each other. Although it's not obvious, for example, that there is any actual meat between turkey and duck, there is nothing impossible about the 
existence of a meat which is just as dissimilar to turkey as beef is to venison - say, for example, turducken - occupying that position.

But if comparative dissimilarity is a relation between possible particulars, then the Archimedean condition is not satisfied, since there is nothing impossible about particulars so dissimilar that no concatenation of ordinary dissimilarities can reach them. It is possible, for example, that there is an infinite library, which contains all finite books. If the Archimedean condition is met, then there should be a finite sequence of libraries, each successive pair of which differs by only one book, such that the first library in the sequence is my library and the last is the infinite library.

But since my library has only finitely many books, each finite sequence of libraries beginning with my library, and each successive pair of which differs by only one book, can only end in a library containing finitely many books - I cannot obtain an infinite library by purchasing only finitely many books. Moreover, the infinite library containing all finite books is just one amongst many possible libraries - there are $2^{\aleph_{0}}$ possible libraries corresponding to every combination of the $\aleph_{0}$ finite books, and more corresponding to combinations of infinite books.

Whereas in the context of an ordinal scale the assumption of countable order-density appeared to be merely a harmless idealisation, in the context of a ratio scale the assumption of the Archimedean condition involves a serious disanalogy between distance and dissimilarity. There is no distance so great that it is not a large but finite multiple of an arbitrarily small distance. But there are possible dissimilarities so great that they are not a finite multiple, no matter how large, of an arbitrarily small dissimilarity. The Archimedean condition is not a harmless idealisation, but a serious limitation.

Two objections. Firstly, whereas (5) the conditions of a proximity structure and (6) countable order-density are jointly necessary and sufficient for representations derived from an ordinal scale, the conditions of a segmentally additive proximity structure are sufficient for representation by a ratio 
scale, but not necessary. So it is possible for a proponent of the analogy between distance and dissimilarity to argue that comparative dissimilarity between actual particulars is representable by a ratio scale, without defending segmental solvability.

Suppose, for example, that the set $A$ contains just four particulars: $a, b$, $c$ and $d$. And suppose $a d \succ b d \succ a c \sim c d \succ a b \sim b c$ (imagine a line with $c$ half way between $a$ and $d$, and $b$ half way between $a$ and $c$ ). Then it follows from (7), the qualitative definition of betweenness, that $c$ is between $a$ and $d$ as well as between $b$ and $d, b$ is between $a$ and $c$ as well as between $a$ and $d, c$ is between $b$ and $d$ as well as between $a$ and $d$, but nothing is between $c$ and $d$. In this case, condition (1), segmental solvability, is not satisfied, since, for example, $c d \succsim a b$, but there is no $e$ such that $c e \sim a b$ and $e$ is between $c$ and $d$.

Nevertheless, $\langle A, \succsim\rangle$ is representable by a distance function $\delta$ according to which $\delta(a, b)=\delta(b, c)$, since $b$ is as far from $a$ as $c$ is from $b, \delta(c, d)=$ $\delta(a, c)=\delta(a, b)+\delta(a, c)=2 \delta(a, b)$, since $b$ is equidistant between $a$ and $c$, $\delta(a, d)=\delta(a, c)+\delta(c, d)=2 \delta(a, c)=4 \delta(a, b)$, since $c$ is equidistant between $a$ and $d$, and $\delta(b, d)=\delta(b, c)+\delta(c, d)=3 \delta(a, b)$, since $c$ is between $b$ and $d$. These distances will be in the same ratios according to any distance function which represents $b$ as equidistant between $a$ and $c$ and $c$ as equidistant between $a$ and $d$. So $\langle A, \succsim\rangle$ is representable by a ratio scale, even though it does not satisfy segmental solvability.

But not every failure of segmental solvability is so favourable. Suppose, for example, that $A$ contains just three particulars: $a, b$ and $c$. And suppose $a c \succ a b \succ b c$ (imagine a line with $b$ between $a$ and $c$, but closer to $c$ than to a). Then it follows from (7), the qualitative definition of betweenness, that $b$ is between $a$ and $c$. And in this case, condition (1), segmental solvability, is still not satisfied, since $a b \succsim b c$, but there is no $e$ such that $a e \sim b c$ and $e$ is between $a$ and $b$. 
In this case $\langle A, \succsim\rangle$ is representable by a distance function $\delta$ according to which $\delta(a, c)>\delta(a, b)>\delta(b, c)$ and, since $b$ is between $a$ and $c, \delta(a, c)=$ $\delta(a, b)+\delta(b, c)$. But these distances will not be in the same ratios according to every distance function which represents $b$ as between $a$ and $c$, since there is not enough information to determine how much closer $b$ is to $c$ than $b$ is to $a$. If the distance between $a$ and $c$ is one, for example, then the respective distances between $a$ and $b$ and $b$ and $c$ could be two thirds and one third, so that $b$ is twice as far from $a$ as from $c$, or three quarters and one quarter, so that $b$ is thrice as far from $a$ as from $c$.

So if segmental solvability is not satisfied, comparative dissimilarity is representable by a ratio scale only under favourable circumstances. But if comparative dissimilarity is a relation between actual particulars, then it's implausible that favourable circumstances obtain, for the same reasons as segmental solvability is implausible. At best, the representability of comparative dissimilarity by a ratio scale would be a lucky accident. But if the analogy between distance and dissimilarity is correct, then that comparative dissimilarity is representable by a ratio scale should not be contingent, but necessary (see [11] for a related point about physical measurement).

Secondly, it might be objected that the field of comparative dissimilarity need not be extended to all possible particulars, but to just those possible particulars which are required in order to satisfy segmental solvability, and not to those possible particulars which would violate the Archimedean condition. The comparative dissimilarity between turkey and duck can be compared to that between beef and venison, for example, by a comparison with turducken, without requiring that these comparisons be extended to bunyips and dragons as well.

But this response would also make the representability of comparative dissimilarity by a ratio scale merely contingent. If the infinite library containing all finite books actually existed, for example, then although a ratio scale could represent the degree of dissimilarity of all its finite subsections 
to each other, a ratio scale could not represent the degree of dissimilarity of any of its finite sections to the whole library. Physical space, in contrast, can be represented on a ratio scale, regardless of whether it is finite or infinite in extent.

Even if the infinite library containing all finite books actually existed, perhaps it may be excluded for the purpose of representing the relation of comparative dissimilarity between its finite subsections by a ratio scale. But even this concession would reveal a substantive disanalogy between distance and dissimilarity. There is no possible distance so great that it is not a large but finite multiple of an arbitrarily small distance. But there are possible dissimilarities so great that they are not a finite multiple, no matter how large, of an arbitrarily small dissimilarity. Dissimilarity is different from distance.

\section{Completeness}

In the last section, I argued that there is a dilemma for the representation of degree of dissimilarity by a ratio scale, because the condition of segmental solvability is not necessarily met by actual particulars, but the Archimedean condition is necessarily not met by possible particulars. In this section I point out the representation of degree of dissimilarity by a ratio scale has a second problem with infinity, which arises from the fact that the conditions of a segmentally additive proximity structure still do not suffice to capture the whole spirit of the analogy between distance and dissimilarity.

The second problem is that the conditions of a segmentally additive proximity structure still do not capture the analogy between distance and dissimilarity, since they still do not suffice to entail that degree of dissimilarity is representable by a dense and complete metric space - in fact, they do not even suffice to entail that there is any irrational degree of dissimilarity. To see this, suppose there is a pair of least dissimilar particulars, or a minimum positive degree of dissimilarity. Then segmental solvability and the 
Archimedean condition entail that between every pair of dissimilar particulars is a finite sequence of least dissimilar particulars, or successive pairs of particulars which are dissimilar to just that degree.

It follows from clause (3), the additivity of the scale, in theorem (2) that the degree of dissimilarity between any two particulars is simply the number of these least dissimilar particulars multiplied by this minimum positive degree of dissimilarity or, in other words, simply proportional to the number of pairs of least dissimilar particulars between them. Supposing that there is only quail and turkey between chicken and duck, for example, it would follow that the degree of dissimilarity between chicken and turkey is simply the minimum distance multiplied by three (for the three pairs, chicken and quail, quail and turkey, and turkey and duck).

And if the scale is chosen so that the minimum positive degree of dissimilarity is one, then it follows that the degree of dissimilarity between any two particulars is simply the number of pairs of least dissimilar particulars between them. In other words, it follows not only that the degree of dissimilarity between any two different particulars is rational, but that it is an integer. Supposing that there is only quail and turkey between chicken and duck, for example, it would follow that the degrees of dissimilarity between chicken and quail, chicken and turkey, and chicken and duck are respectively one, two and three.

So in order to fully capture the analogy between distance and dissimilarity, it's desirable to add further qualitative conditions than those of a segmentally additive proximity structure. The following definition gives qualitative conditions which not only suffice for degree of dissimilarity to be represented by a distance function which delivers irrational values, but also defines a dense and complete metric space:

Definition 9. [13, 168] A segmentally additive proximity structure is complete if and only if for all distinct $a, b \in A$ :

(1) There exist distinct $c, d \in A$ such that $a b \succ c d$ (nonminimality) 
(2) For all distinct $c, d \in A$ and every sequence $e_{1}, \ldots, e_{n}, \ldots$ of elements of $A$, if there is an $m$ such that $c d \succsim e_{i} e_{i+1}$ for all $i>m$, then there is an $e \in A$ such that for all distinct $f, g \in A$ there is an $l$ such that $f g \succsim a_{i} e$ for all $i>l$ (qualitative completeness)

If particulars under the relation of comparative dissimilarity, for example, are a complete segmentally additive structure, then that suffices for some degrees of dissimilarity to be irrational.

If comparative dissimilarity meets condition (1), nonminimality, then every pair of different particulars is strictly less dissimilar than some other pair of different particulars - no matter how similar venison is to beef, for example, there must be some pair - say, chicken and turkey - which are even more similar. Nonminimality entails that there is no minimum positive distance or degree of dissimilarity - since if some pair were that minimum positive distance apart, there would be a dissimilar pair which are less dissimilar, and so (impossibly) a positive distance less than the positive minimum apart. So nonminimality entails that not all degrees of dissimilarity are integers.

But even though it entails that there is no no minimum positive degree of dissimilarity, nonminimality still does not entail that any degree of dissimilarity is irrational, since it is compatible with the conditions given so far that every degree of dissimilarity is rational. The rational numbers $\mathbb{Q}$ under the quaternary relation $i j \succsim k l$ if and only if $|i-j| \geq|k-l|$, for example, meet all the conditions of a segmentally additive proximity structure, and so are represented by the distance function corresponding to their absolute difference $\delta(i, j)=|i-j|$. But although this distance function has no positive minimum, it does not take irrational values.

So in order to entail that some degrees of dissimilarity are irrational, condition (4), completeness, is also required. According to it, if the successive pairs in a sequence of particulars become arbitrarily similar to one another, then there is one particular in particular which the sequence of particulars becomes arbitrarily similar to. If my paternal ancestors, for example, 
evolved so that for any two people, there was a generation before which every father was more similar to his son than the two people to each other, then completeness requires that there is an archetypal person such that for any other person, there was a generation before which all my paternal ancestors were more similar to the archetypal person than to that other person.

In the context of segmental solvability, condition (1) of the definition of a segmentally additive proximity structure, nonminimality corresponds to the condition of density on the corresponding metric space. In other words, since for any two distinct points nonminimality entails that there are two distinct points closer together, and segmental solvability entails that there are points in between the first two points and the distance of the closer points apart, nonminimality and segmental solvability jointly entail that between any two distinct points there is a third.

Likewise, the completeness condition (2) of definition (9) is simply a qualitative version of the quantitative completeness condition provided earlier in definition (4). So the conditions of a complete segmentally additive proximity structure suffice to entail that every two points in the metric space described in theorem (2) are joined by a metric segment or, in other words, by a subset of the space isometric to a Euclidean line segment. Moreover, they thus suffice to capture the analogy between distance and dissimilarity.

Since the metric segment between two points is isometric to a Euclidean line segment of the same length, corresponding to each irrational length on the Euclidean line segment is an irrational distance on the metric segment, and so the conditions of a complete segmentally additive proximity structure also suffice to entail that some degrees of dissimilarity are irrational. So whereas the definition of a segmentally additive proximity structure is compatible with all degrees of dissimilarity being integers, the conditions of nonminimality and completeness suffice for degrees of dissimilarity being fractional and irrational respectively. 
But although this resolves the second problem with infinity, it exacerbates the first. Like the condition of segmental solvability, the condition of nonminimality is not necessary for actual particulars - it is possible that some pair of dissimilar particulars are as similar to each other as any pair of dissimilar particulars are. This is particularly obvious if the number of actual particulars is finite, in which case there must be a minimum positive degree of dissimilarity, simply because every finite subset of positive real numbers has a minimum.

But even if the number of actual particulars is not finite, they might have been finite. So once again, the representability of comparative dissimilarity by distance in a complete metric space would be a lucky accident. But as I argued in the last section, if the analogy between distance and dissimilarity is correct, then that comparative dissimilarity is representable by distance in a complete metric space should not be be merely contingent, but necessary. So the condition of nonminimality motivates interpreting comparative dissimilarity as a relation between possible particulars.

If comparative dissimilarity is interpreted as a relation between actual particulars, and the number of actual particulars is finite, then the completeness condition is vacuously fulfilled. But if comparative dissimilarity is interpreted as a relation between possible particulars then completeness is not satisfied. Suppose, for example, that each of my paternal ancestors were half as tall as the last, in which case for any two people, there was a generation before which each successive pair of my paternal grandfathers were more similar to each other in respect of height than those two people.

If (4) completeness is met, then there should be an archetypal person such that for any other person, there was a generation before which each of my paternal ancestors were more similar in respect of height to the archetypal person than to that other person. But if the archetypal person had a positive height, then there would be a generation after which all my paternal ancestors are more than half as short as the archetypal person, and so more 
similar to each other than they are to the archetypal person. It follows that the archetypal person cannot have a positive height - which is impossible.

So the dilemma for the thesis that degree of dissimilarity is representable by distance in a metric space is exacerbated. If comparative dissimilarity is a relation between actual particulars, then although it meets the condition of completeness (2), it may not meet the condition of nonminimality (1). But if comparative dissimilarity is a relation between possible particulars, then although it meets the condition of nonminimality (1), it does not meet the condition of completeness (2). In either case, the analogy between distance and dissimilarity is lost.

\section{Conclusion}

The thesis that degree of dissimilarity is distance in a metric space faces a dilemma. On the one hand, comparative dissimilarity satisfies, but for a technicality, the qualitative conditions required for a representation as distance in a metric space to be derived from an ordinal scale. But since the resulting distances may be rescaled arbitrarily, a representation derived from an ordinal scale does not suffice to capture the analogy between distance and dissimilarity. If dissimilarity is truly analogous to distance, it should be represented by a ratio scale, which would represent not only which things are more similar to each other, but also by how much.

On the other hand, the relation of comparative dissimilarity does not satisfy the qualitative conditions required for representation by a ratio scale, regardless of whether it is construed as a relation between actual or possible particulars. If comparative dissimilarity is considered as a relation between actual particulars, then it may not satisfy the condition of segmental solvability, since not all dissimilar actual particulars must have actual particulars between them at the relevant intervals.

But if comparative dissimilarity is considered as a relation between possible particulars, then although it satisfies segmental solvability, it does not 
satisfy the Archimedean condition, since there are some possible particulars which are so dissimilar that their dissimilarity does not divide into any finite number of small dissimilarities. So whether comparative dissimilarity is interpreted as a relation between possible or actual particulars, it is not representable by a ratio scale either way.

The problem is exacerbated by conditions required to ensure that degree of dissimilarity be representable by a complete metric space. If comparative dissimilarity is considered as a relation between actual particulars, then the condition of nonminimality is not met, since it's plausible that there are two dissimilar particulars such that no dissimilar particulars are less dissimilar. But if comparative dissimilarity is considered as a relation between possible particulars, the condition of completeness is not met, exacerbating the original dilemma.

So the traditional justification of the thesis that degree of dissimilarity is distance in a metric space - via the proof of a representation and uniqueness theorem, according to which if comparative dissimilarity meets certain qualitative conditions, then it is uniquely representable by distance in a metric space - fails, since comparative dissimilarity does not meet conditions which are strong enough to justify the analogy between distance and dissimilarity. The analogy between distance and dissimilarity is suggestive, but unsuccessful 1

\section{REFERENCES}

[1] John Bigelow. Possible Worlds Foundations for Probability. Journal of Philosophical Logic, 5(3):299-320, August 1976.

[2] John Bigelow. Semantics of Probability. Synthese, 36(4):459-472, December 1977.

\footnotetext{
${ }^{1}$ Thanks to Edward Elliot, Alan Hajek, Cliff Kerr, Daniel Nolan, Michael Pelczar, Alex Sandgren and Neil Sinhababu, as well as audiences at the Australian National University and the University of Hong Kong for discussion of this paper.
} 
[3] Leonard Blumenthal. Theory and Applications of Distance Geometry. Oxford University Press, Oxford, 1953.

[4] John Keynes. A Treatise on Probability. MacMillan, London, 1921.

[5] David Krantz, Duncan Luce, Patrick Suppes, and Amos Tversky. Foundations of Measurement, Vol. I: Additive and Polynomial representations. Academic Press, San Diego, 1971.

[6] Thomas Kroedel and Franz Huber. Counterfactual Dependence and Arrow. Nous, 47(3):453-466, September 2013.

[7] David Lewis. Counterfactuals. Blackwell, Oxford, 1973.

[8] Michael Morreau. It Simply Does Not Add Up: Trouble with Overall Similarity. Journal of Philosophy, 107(9):469-490, 2010.

[9] Ilkka Niiniluoto. Truthlikeness. Kluwer, Dordrecht, March 1987.

[10] Graham Oddie. Likeness to Truth. Kluwer, Dordrecht, 1986.

[11] Zee Perry. Properly Extensive Quantities. Philosophy of Science, 82(5):833-844, December 2015.

[12] Robert Stalnaker. Anti-Essentialism. Midwest Studies In Philosophy, 4(1):343-355, September 1979.

[13] Patrick Suppes, David Krantz, Duncan Luce, and Amos Tversky. Foundations of Measurement, Vol. 2: Geometrical, Threshold, and Probabilistic Representations. Academic Press, San Diego, 1989.

[14] Amos Tversky. Features of Similarity. Psychological Review, 84:327$352,1977$.

[15] Timothy Williamson. First-Order Logics for Comparative Similarity. Notre Dame Journal of Formal Logic, 29(4):457-481, 1988. 